\title{
Influência da Cobertura Dielétrica Extra no Comportamento dos Modos de Plasmon em Fibras Fracamente Guiadas.
}

\author{
Antonio Romeiro Sapienza e Ricardo Gomes da Costa
}

\begin{abstract}
Resumo - Neste artigo, são analisados os modos de Plasmon em fibras ópticas fracamente guiadas em estruturas com quatro regiões. A análise com a região extra dielétrica, sobre o filme metálico, foi essencial para se compreender o comportamento dos quatro modos de Plasmon; os ligados simétrico (Sb) e assimétrico (ab); fuga pelo núcleo (ln) e fuga pela cobertura (lc). Os modos de Plasmon são, fundamentados no modo TM01. A estrutura analisada é constituída por um filme metálico $(\mathbf{G m})$, rodeado pelo núcleo $(\eta 1)$, e pela cobertura $(\eta 4)$. Entre a cobertura e o filme metálico é depositado um dielétrico extra $(\eta 3)$. São calculadas as curvas de dispersão dos diferentes modos, em função do raio interno da fibra, espessura do filme condutor e da largura da cobertura extra. O método adotado, neste atigo, é validado comparando-se os resultados encontrados, com os do método convencional aplicado a uma estrutura constituida por três regiões.
\end{abstract}

Palavras chave - Modos de Plasmon, modo TM01, equação de Helmholtz cilíndrico-circular, índice efetivo dos respectivos modos, condições de fronteiras entre duas regiões.

Abstract - In this article, the Plasmon modes are analyzed in weakly guided optical fibers in four regions structures. The analysis with the extra dielectric region, on the metallic film, was essential to understand the behavior of the four Plasmon modes; the symmetrical (Sb) and asymmetric bounded (ab); the core (In) and covering leaky modes (lc). The Plasmon modes are, based in the TM01 formulation. The analyzed structure is constituted by a metallic film $(\mathrm{Cm})$, surrounded by the core $(\eta 1)$, and for the covering ( $\eta 4)$. Between the covering and the metallic film an extra dielectric is deposited $(\eta 3)$. The dispersion curves are calculated in function of the fiber internal ray, film thickness and the covering width. The validation method presented in this article is obtained by confronting with the conventional method results applied in an optical fiber with three regions.

Key words - Plasmon Modes, TM01 Formulation, cylindricalcircular Helmholtz equation, respective modes effective index, borders conditions between two areas.

\section{INTRODUÇÃO}

Metais são materiais que apresentam a parte real da

Ricardo Gomes da Costa e Antonio Romeiro Sapienza. DEE - Depto de Engenharia Eletrônica , Universidade do Estado do Rio de Janeiro - UERJ, Rio de Janeiro, RJ, E-mails: ricgoco@gmail.com e a_sapienza@osite.com.br permissividade negativa. Esta característica é devido ao acoplamento do campo eletromagnético incidente à densidade dos elétrons livres da banda de condução do metal. A conseqüência deste acoplamento é o guiamento de ondas evanescentes nas fronteiras entre o metal e os dielétricos que o circundam. Estas ondas são conhecidas por ondas de Plasmon ("plasma" se refere aos elétrons livres da banda de condução do metal, e o sufixo "on" ao substantivo; fóton, partícula). No metal, os elétrons acoplados ao campo eletromagnético incidente oscilam dissipando energia por efeito Joule. Portanto, o modelo eletromagnético dos metais, sob a ação de ondas harmônicas da forma $\left(\mathrm{e}^{+\mathrm{jwt}}\right)$ é caracterizado pela permissividade:

$$
\epsilon_{\mathrm{m}}=-\epsilon_{0}\left(\epsilon_{\mathrm{mr}}+\mathrm{j} \epsilon_{\mathrm{mi}}\right) \quad[1,2,3,4]
$$

Neste artigo, são estudados os modos de Plasmon nas superfícies de um filme condutor $\left(\epsilon_{\mathrm{m}}\right)$ de espessura (d) depositado sobre fibras ópticas fracamente guiadas $\left(\eta_{1}\right)$ de raio (a). O filme condutor é recoberto por uma região dielétrica extra $\left(\eta_{3}\right)$ de espessura $(h)$ que por sua vez faz fronteira com a cobertura $\left(\eta_{4}\right)$ infinita, como mostra a Figura.1.

A abordagem é feita pela técnica clássica de casamento dos campos nas respectivas fronteiras da estrutura.

Os quatro modos de Plasmon são, todos, naturais do modo $\mathrm{TM}_{01}$, que em função do comportamento da componente Real $\left[\mathrm{H}_{\theta}(\mathrm{r}, \mathrm{z})\right]$ são denominados por; modos ligados simétrico e assimétrico $\left(S_{b}\right.$ e $\left.a_{b}\right)$, fuga pela cobertura $\left(l_{c}\right)$ e fuga pelo núcleo $\left(l_{n}\right) \cdot[5]$

Os índices efetivos $\left(\eta \mathrm{ef}_{\mathrm{r}}\right.$ e $\eta \mathrm{f}_{\mathrm{i}}$ ) dos respectivos modos de Plasmon, são analisados em função do raio interno da fibra (a), largura da cobertura (h), variando-se a espessura do filme condutor (d).

Os resultados obtidos mostraram que o modo $1_{c}$ (fuga pela cobertura) é fortemente influenciado pela região dielétrica extra $\left(\eta_{3}\right)$ sobre o filme. Para espessuras normalizadas da cobertura apresentadas até $\mathrm{K}_{0} \mathrm{~h}=10$, o respectivo modo é dependente da região extra $\left(\eta_{3}\right)$.

\section{MODELO MATEMÁTICO}

\section{A. Equação de Helmholtz normalizada.}

A estrutura em fibra óptica fracamente guiada com quatro regiões, é vista na Figura.-1 $(a, b)$. 

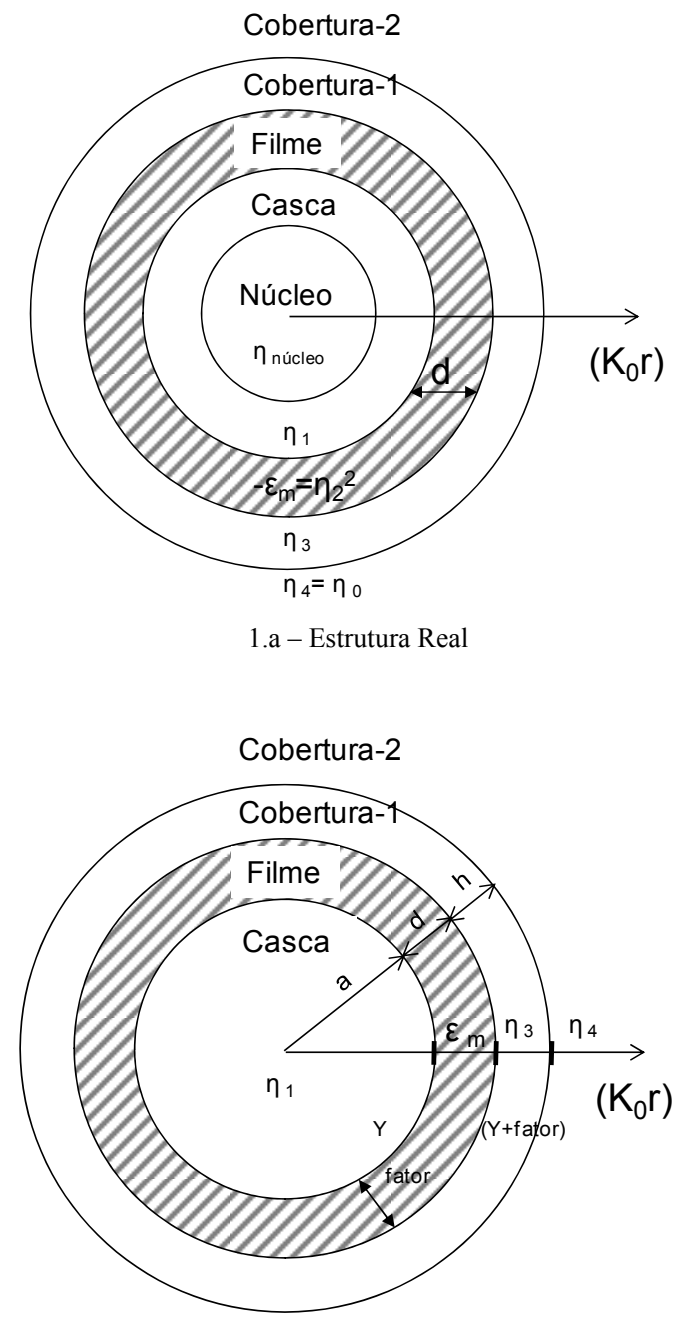

$1 . b$ - Estrutura Equivalente.

Figura.-1 - Fibra óptica fracamente guiada com quatro regiões.

A fibra óptica apresentada na figura-1.a, como é fracamente guiada, a análise dos modos de Plasmon é feita pela estrutura equivalente, mostrada na Figura.-1.b. Os modos de Plasmon são modos $\mathrm{TM}_{01}$, com simetria angular e

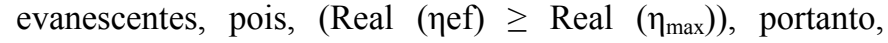
satisfazem em cada região, $\mathrm{i}=(1, \mathrm{~m}, 3,4)$, vide Figura.-1.b, as seguintes equações de Helmholtz em coordenadas cilíndricas circulares:

$r \frac{d}{d r}\left[r \frac{d R_{i}(r)}{d r}\right]-\left(r \alpha_{i}\right)^{2} R_{i}(r)=0 \quad i=(1, m, 3,4)$
$\mathrm{r}=\left(\mathrm{K}_{0} \mathrm{r}\right) \rightarrow$ raio normalizado
$\mathrm{h}=\left(\mathrm{K}_{0} \mathrm{~h}\right) \rightarrow$ espessura normalizada da cobertura.

Cuja solução fornece:

$E_{z i}(r, z)=A R_{i}(r) e^{-j \beta z} \quad i=(1, m, 3,4)$
A equação (1) é a adequada ao cálculo dos modos evanescentes nos sistemas circulares, onde:

$$
\begin{array}{ll}
\mathrm{H}_{\theta \mathrm{i}}(\mathrm{r}, \mathrm{z})=\frac{\mathrm{j}}{\mathrm{Z}_{0}}\left[\frac{\left(\eta_{\mathrm{i}}\right)^{2}}{\alpha_{\mathrm{i}}}\right] \frac{\mathrm{dE}_{\mathrm{zi}}}{\mathrm{dr}} & \mathrm{Z}_{0}=\sqrt{\frac{\mu_{0}}{\varepsilon_{0}}}=120 \pi \\
\beta=\mathrm{K}_{0} \cdot \eta \text { ef } & \eta \mathrm{ef}=\eta \mathrm{ef}_{\mathrm{r}}-\mathrm{j} \eta e f_{\mathrm{i}} \quad\left(4^{\mathrm{o}} \text { quadrante }\right) \\
\left.\mathrm{K}_{\mathrm{i}}=\mathrm{K}_{0} \cdot \eta_{\mathrm{i}} \quad \mathrm{i}=(1,3,4) \quad \text { (Dielétrico }\right) \\
\mathrm{K}_{\mathrm{m}}=\mathrm{K}_{0} \cdot \sqrt{-\varepsilon_{\mathrm{rm}}} \quad \varepsilon_{\mathrm{rm}}=\left(\varepsilon_{\mathrm{mr}}+\mathrm{j} \cdot \varepsilon_{\mathrm{mi}}\right) \quad \text { (FilmeMetálico) }
\end{array}
$$

Como são modos evanescentes da estrutura têm-se:

$$
\left(\mathrm{K}_{\mathrm{ti}}\right)^{2}=-\left(\mathrm{K}_{0}\right)^{2} \cdot\left[\eta^{2} \mathrm{f}^{2}-\left(\eta_{\mathrm{i}}\right)^{2}\right]
$$

Consequentemente:

$\begin{array}{ll}\mathrm{K}_{\mathrm{ti}}=\mathrm{j} \cdot \mathrm{K}_{0} \cdot \alpha_{\mathrm{i}} & \alpha_{\mathrm{i}}=\sqrt{\mathrm{nef}^{2}-\left(\eta_{\mathrm{i}}\right)^{2}} \quad \text { (Dielétricos) } \\ \mathrm{K}_{\mathrm{tm}}=\mathrm{j} \cdot \mathrm{K}_{0} \cdot \alpha_{\mathrm{m}} & \alpha_{\mathrm{m}}=\sqrt{\mathrm{nef}^{2}+\varepsilon_{\mathrm{rm}}} \quad \text { (FilmeMetálico) }\end{array}$

A equação (1) aplicada à Figura.-1.b, apresenta as seguintes soluções: Considere o raio normalizado $\left(\mathrm{K}_{0} \mathrm{r}\right)=\mathrm{r}$.

Região dielétrica $\left(\eta_{4}\right): R_{4}\left(\alpha_{4} r\right)=A_{4} F_{40}\left(\alpha_{4} r\right)$

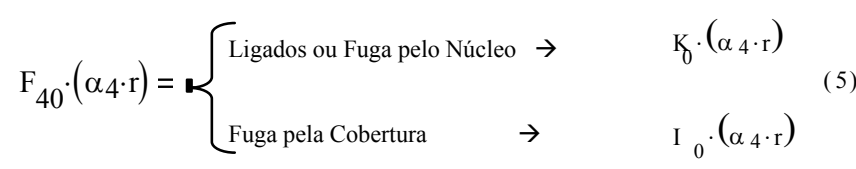

Cobertura extra $\left(\eta_{3}\right): R_{3}\left(\left(K_{0} r\right) \alpha_{3}\right)=A_{3} K_{0}\left(\alpha_{3} r\right)+B_{3} I_{0}\left(\alpha_{3} r\right)$

Filme metálico $\left(\epsilon_{m}\right): R_{m}\left(\left(\alpha_{m} r\right)=A_{m} K_{0}\left(\alpha_{m} r\right)+B_{m} I_{0}\left(\alpha_{m} r\right)\right.$

Região do núcleo $\left(\eta_{1}\right): R_{1}\left(\alpha_{1} r\right)=A_{1} F_{10}\left(\alpha_{1} r\right)$

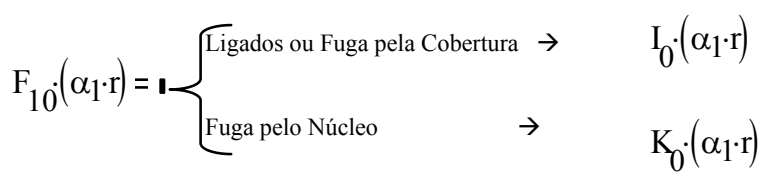

Os resultados desta análise são, portanto correspondentes aos valores normalizados $\mathrm{r}=\left(\mathrm{K}_{0} \mathrm{r}\right)$.

B. Cálculo das componentes dos campos eletromagnéticos tangenciais as fronteiras.

Os campos elétricos e magnéticos tangentes às respectivas fronteiras da estrutura, Figura.-1.b, são $\left(\mathrm{E}_{\mathrm{zi}}, \mathrm{H}_{\theta \mathrm{i}}\right)$, que com auxílio de (1) se escrevem. 


\begin{tabular}{cl|}
\hline$r \geq(a+d+h) K_{0}$ & $E_{z 4}(r)=A_{4} F_{40}\left(\alpha_{4} r\right)$ \\
$(a+d+h) K_{o} \geq r \geq(a+d) K_{o}$ & $E_{23}(r)=A_{3} K_{0}\left(\alpha_{3} r\right)+B_{3} I_{0}\left(\alpha_{3} r\right)$ \\
$(a+d) K_{0} \geq r \geq a K_{0}$ & $E_{z m}(r)=A_{m} K_{0}\left(\alpha_{m} r\right)+B_{m} I_{0}\left(\alpha_{m} r\right)$ \\
$r \leq a K_{0}$ & $E_{z 1}(r)=A_{1} F_{10}\left(\alpha_{1} r\right)$ \\
\hline
\end{tabular}

Onde: $\quad F 40(\alpha 4$ r) e F10 ( $\alpha 1$ r) são dados em (5) e (6).

Os campos magnéticos são:

$$
\begin{array}{ll}
\mathrm{r} \geq(\mathrm{a}+\mathrm{d}+\mathrm{h}) \mathrm{K}_{\mathrm{o}} & H_{\theta i}(r)=\frac{j A_{4}}{Z_{0}}\left[\frac{\eta_{4}^{2}}{\alpha_{4}}\right] F_{41}\left(\alpha_{4} r\right) \\
(\mathrm{a}+\mathrm{d}+\mathrm{h}) \mathrm{K}_{\mathrm{o}} \geq \mathrm{r} \geq(\mathrm{a}+\mathrm{d}) \mathrm{K}_{\mathrm{o}} & H_{\theta i}(r)=\frac{j}{Z_{0}}\left[\frac{\eta_{3}^{2}}{\alpha_{3}}\right]\left[-A_{3} K_{1}\left(\alpha_{3} r\right)+B_{3} I_{1}\left(\alpha_{3} r\right)\right] \\
\mathrm{r} \leq \mathrm{a} \mathrm{K}_{\mathrm{o}} & H_{\theta i}(r)=\frac{j A_{1}}{Z_{0}}\left[\frac{\eta_{1}^{2}}{\alpha_{1}}\right] F_{11}\left(\alpha_{1} r\right)
\end{array}
$$

Onde:

$$
F_{41}\left(\alpha_{4} r\right)= \begin{cases}\text { Ligados ou fuga pelo núcleo } & \rightarrow-K_{l}\left(\alpha_{4} r\right) \\ \text { Fuga pela cobertura } & \rightarrow I_{l}\left(\alpha_{4} r\right)\end{cases}
$$

e

$F_{11}\left(\alpha_{1} r\right)= \begin{cases}\text { Ligados ou fuga pela cobertura } & \rightarrow I_{1}\left(\alpha_{1} r\right) \\ \text { Fuga pelo núcleo } & \rightarrow-K_{l}\left(\alpha_{1} r\right)\end{cases}$

C. Cálculo da equação característica dos modos de Plasmon.

A equação característica dos modos de Plasmon, Figura.1.b, é obtida pelo casamento dos campos nas respectivas fronteiras normalizadas, ou seja:

Denominando:

$$
\begin{aligned}
& \mathrm{r}_{2}=(\mathrm{a}+\mathrm{d}+\mathrm{h}) \mathrm{K}_{\mathrm{o}} \\
& \mathrm{r}_{2}=(\mathrm{a}+\mathrm{d}) \mathrm{K}_{\mathrm{o}} \quad r_{i}=\frac{n_{i}^{2}}{\alpha_{i}} \quad i=(1, m, 3,4) \text { vide eq. } \\
& \mathrm{r}_{\mathrm{a}}=\mathrm{a} \mathrm{K} \mathrm{K}_{\mathrm{o}}
\end{aligned}
$$

Resulta no sistema matricial:

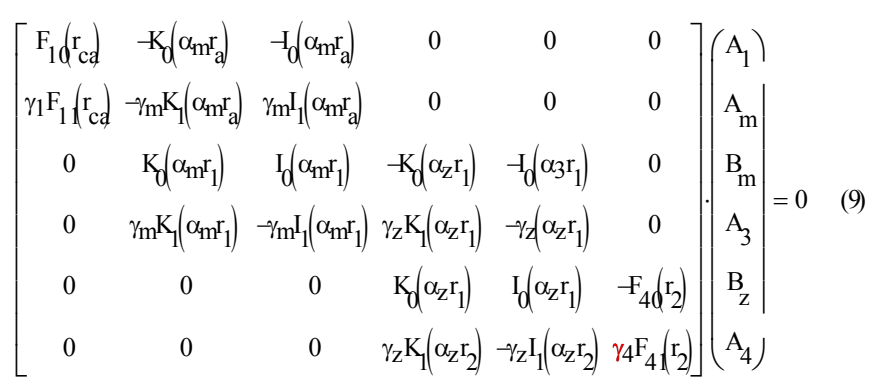

A solução procurada é o valor de $\eta e f=\left(\eta e f_{r}-j \eta f_{i}\right)$ que anula o determinante da matriz (9). Trata-se, portanto, de um problema relacionado a duas equações transcendentais, não lineares:

Real $[$ det $]=0$

Imag $[\mathrm{det}]=0$

\section{RESULTADOS}

Os resultados, originais, apresentados, neste trabalho, são obtidos com a participação do dielétrico extra, sobre o filme metálico, vide Figura.-1.b.

A presença deste dielétrico esclareceu a dependência dos respectivos modos de Plasmon com os meios dielétricos fronteiriços ao filme condutor, evidenciando, que o modo de fuga pela cobertura depende da espessura do dielétrico extra $\left(\eta_{\mathbf{a}}\right)$ em contato com a face externa do filme. Enquanto que os modos ligados e o de fuga pelo núcleo não sofrem, qualitativamente, influencia da espessura do dielétrico ( $\eta 3$ ) sobre o filme condutor.

A estrutura analisada, Figura. 1.b, é constituída por uma fibra de raio normalizado $(\mathrm{K} 0 \mathrm{a})=8$ e $\eta 1=1.515$. O filme metálico utilizado foi a prata, com $€ \mathrm{rm}=19+\mathrm{j} 0.53$, variandose (K0d) de 0 a 1 . A cobertura extra, $\eta 3=1.5$, de espessura normalizada (K0h) assumiu valores entre 0.001 e 10 , o valor de $(\mathrm{K} 0 \mathrm{~h})=10$ é considerado infinito. A região da cobertura da estrutura, $\eta 4=1,53$, é considerada de extensão infinita.

A análise tem início pelos cálculos dos valores

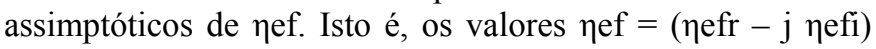
quando o raio do núcleo da fibra tende ao infinito $(\mathrm{a} \rightarrow \infty)$. Os valores assimptóticos dependem exclusivamente das regiões dielétricas fronteiriças ao filme condutor $(\eta 1=1.515 \mathrm{e}$ $\eta 3=1.5)$, de acordo com $[5,6]$.

$n e f_{r}($ assimptótico $)=\sqrt{\varepsilon_{p}} \frac{\sqrt{\varepsilon_{m r}^{2}-\varepsilon_{m} \varepsilon_{p}+\varepsilon_{m i}^{2}}}{\left|\varepsilon_{m}-\varepsilon_{p}\right|}$

$n e f_{i}($ assimptótico $)=\frac{1}{2 n e f_{r}} \frac{\varepsilon_{m i} \varepsilon_{p}^{2}}{\left|\varepsilon_{m}-\varepsilon_{p}\right|}$

Onde $\varepsilon_{m}=\left(\varepsilon_{m r}+j \varepsilon_{m i}\right)$ permissividade do filme condutor $\varepsilon_{p} ; \mathrm{p}=1$ região do núcleo e $\mathrm{p}=3$ região da cobertura extra.

Os valores assimptóticos da estrutura com o filme de prata analisada neste trabalho, calculados por (10) são:

$$
\begin{aligned}
& n e f=1,597-j 2,99 \times 10^{-3}(\text { solução-1 }) \\
& n e f=1,616-j 3,053 \times 10^{-3}(\text { solução- } 2)
\end{aligned}
$$

Com estes valores assimptóticos de $\eta$ ef foram obtidas as

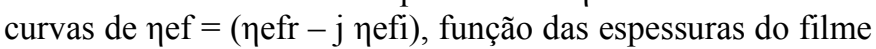
(d) e da cobertura (h) mostradas nos gráficos 1, 2 e 3

As análises dos modos Simétricos e Assimétricos ligados ( $\mathrm{Sb}$ e ab) são mostradas nos gráficos la e $1 \mathrm{~b}$. O

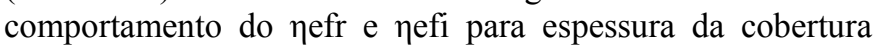


extra ( $\eta 3)$ de 0,01 a 2,44, são vistos nos gráficos 1.a e 1.b, enquanto que os de $\mathrm{h}$ superiores a 4, são mostrados nos gráficos 1c e 1d. Estes resultados são semelhantes aos encontrados na análise das estruturas convencionais de três regiões [4,7]. O que caracteriza a não influência da cobertura extra sobre os referidos modos.

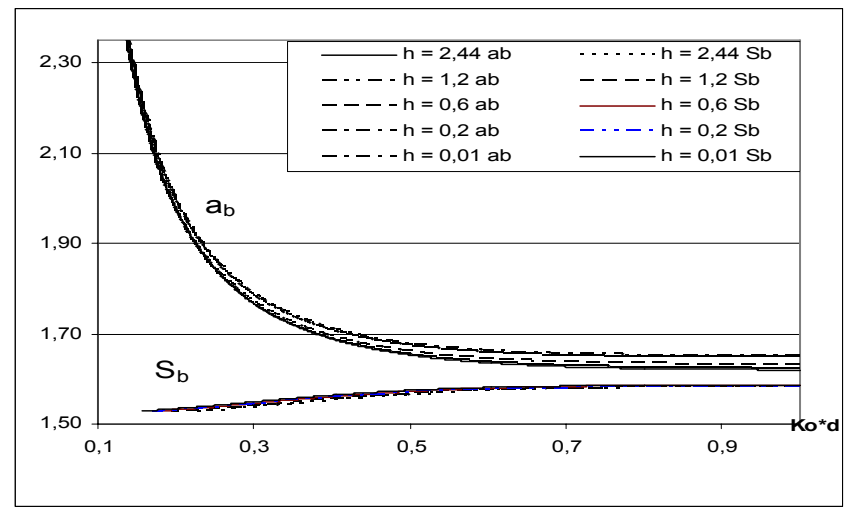

Grafico $1 \mathrm{a}-\left(\eta \mathrm{f}_{\mathrm{r}} * \mathrm{~K}_{\mathrm{o}} \mathrm{d}\right)$ função de $\mathrm{h}$. Os modos $\left(\mathrm{S}_{\mathrm{b}}\right.$ e $\left.\mathrm{a}_{\mathrm{b}}\right)$ se comportam de forma convencional.

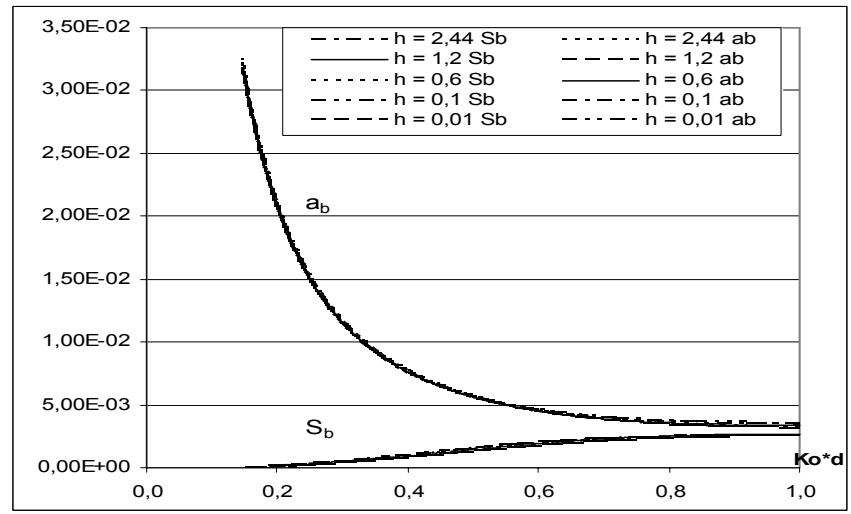

Gráfico $1 b-\left(\eta f_{i} \times K_{0} d\right)$ função de $h$. Os modos $\left(S_{b}\right.$ e $\left.a_{b}\right)$ se comportam de forma convencional.

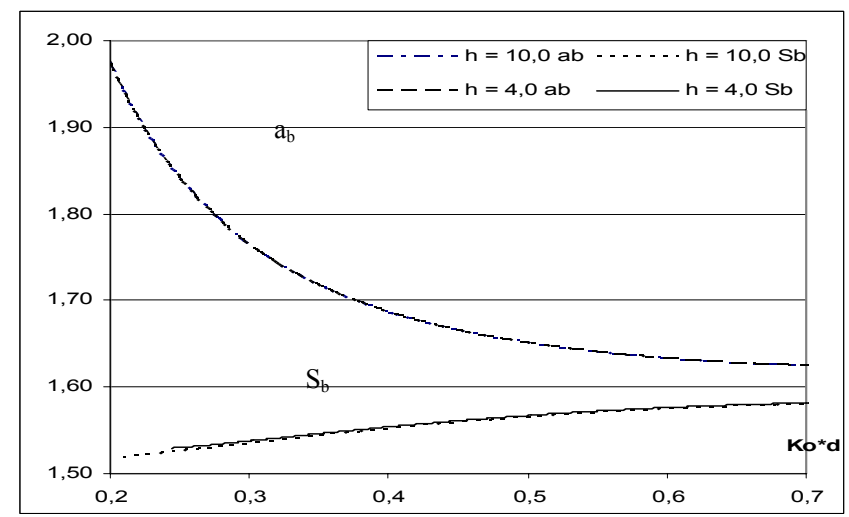

Gráfico $1 \mathrm{c}-\left(\eta \mathrm{f}_{\mathrm{r}} * \mathrm{~K}_{\mathrm{o}} \mathrm{d}\right)$ função de $\mathrm{h}$. Os modos $\left(\mathrm{S}_{\mathrm{b}}\right.$ e $\left.\mathrm{a}_{\mathrm{b}}\right)$ se comportam de forma convencional.

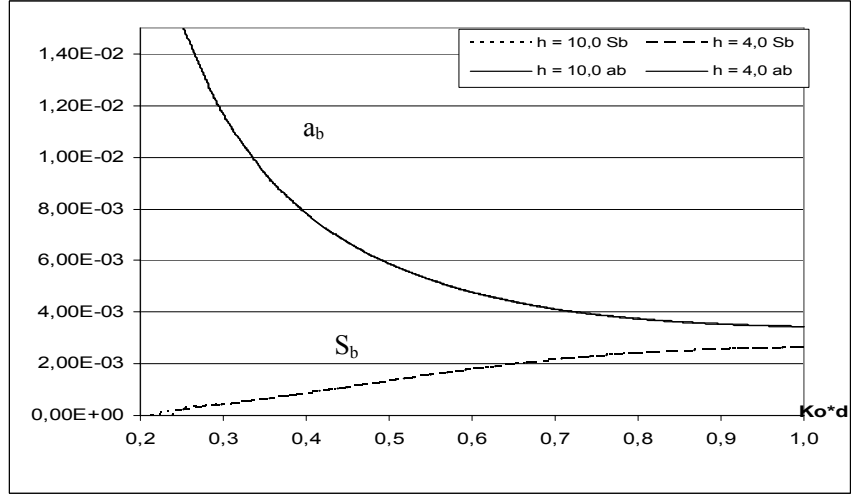

Gráfico $1 \mathrm{~d}-\left(\eta \mathrm{fe}_{\mathrm{i}} \times \mathrm{K}_{\mathrm{o}} \mathrm{d}\right)$ função de $\mathrm{h}$ Os modos $\left(\mathrm{S}_{\mathrm{b}}\right.$ e $\left.\mathrm{a}_{\mathrm{b}}\right)$ se comportam de forma convencional.

A análise dos modos de fuga pela cobertura (lc) é mostrada

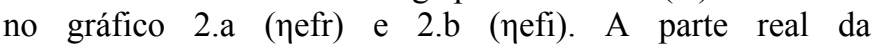

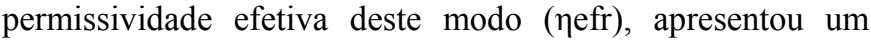
comportamento diferente do convencional, conforme mostrada no gráfico 2.a, referente a estrutura de três regiões, pois, o ๆefr deste modo, é crescente com a diminuição de (K0d). Comprova-se, portanto a influência da cobertura extra ( $\eta 3)$ em contato com o filme condutor, no modo de fuga pela cobertura, alterando a característica do nefr deste modo, tornando o decrescente. O que leva a crer que, a energia evanescente se concentra somente na região dielétrica sobre o filme condutor não incidindo na região ( 4 4), consequentemente, descaracterizando a fuga pela cobertura. $\mathrm{O}$ ๆefi, deste modo, é apresentado no gráfico 2.b. Este parâmetro é, qualitativamente, idêntico ao das estruturas constituídas por três regiões.

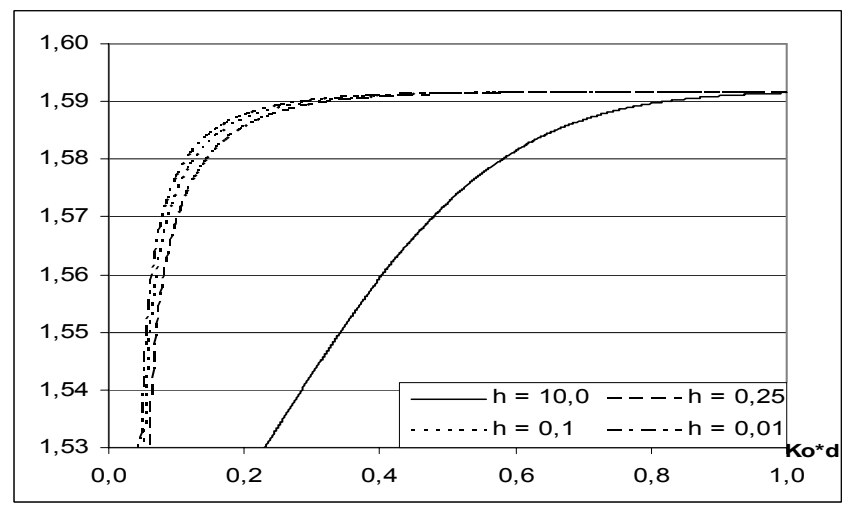

Grafico 2.a - $\left(\eta e f_{r} \times K_{o} d\right)$ função de h do modo fuga pela cobertura ( $\left.1_{c}\right)$.

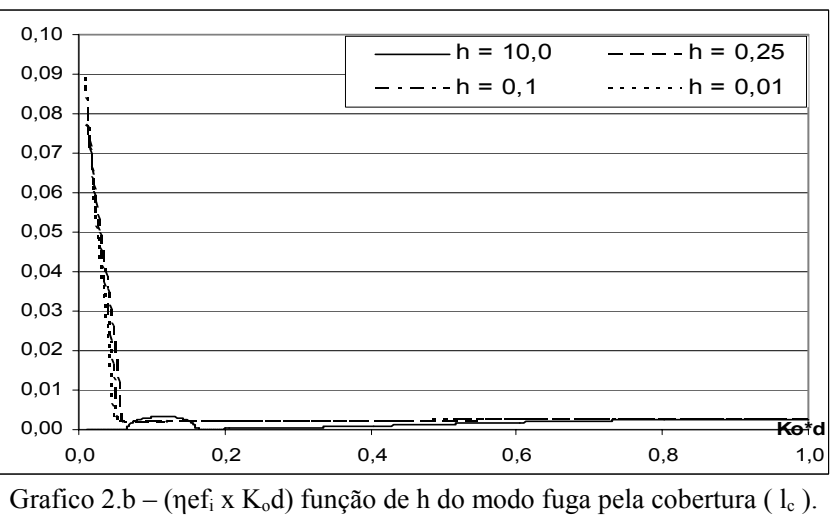


Os modos de fuga pelo núcleo (ln) são analisados nos

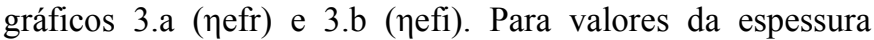
normalizada entre 0.01 e 0.1 , a permissividade efetiva do

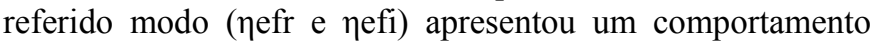
similar ao da estrutura constituída por três regiões [4,7]. Para valores de espessura superiores a 4.0, os parâmetros da permissividade efetiva se comportaram conforme o gráfico 3.c e 3.d, também coerenre com a estrutura clássica de três regiões. Por conseguinte, para quaisquer espessuras do dielétrico extra, estes parâmetros não sofrem a influência da respectiva região extra sobre o filme condutor.

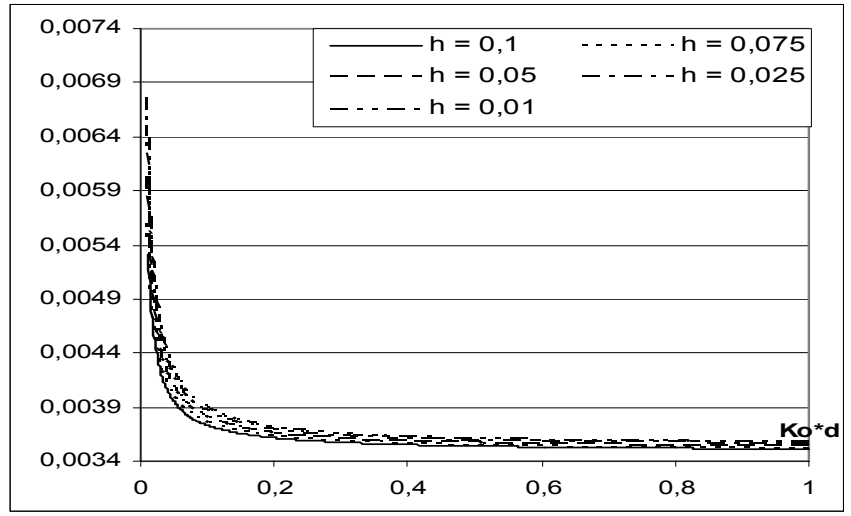

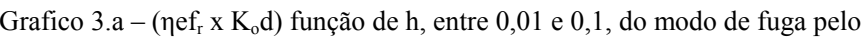
núcleo $\left(1_{n}\right)$.

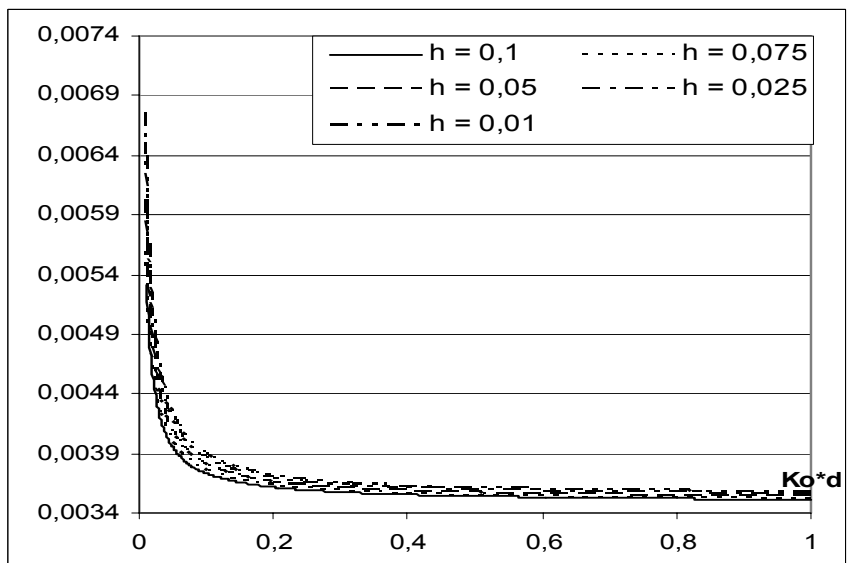

Grafico 3.b - $\left(\eta \mathrm{f}_{\mathrm{i}} \times \mathrm{K}_{\mathrm{o}} \mathrm{d}\right)$ função de $\mathrm{h}$, entre 0,01 e 0,1 , do modo de fuga pelo núcleo $\left(l_{n}\right)$.

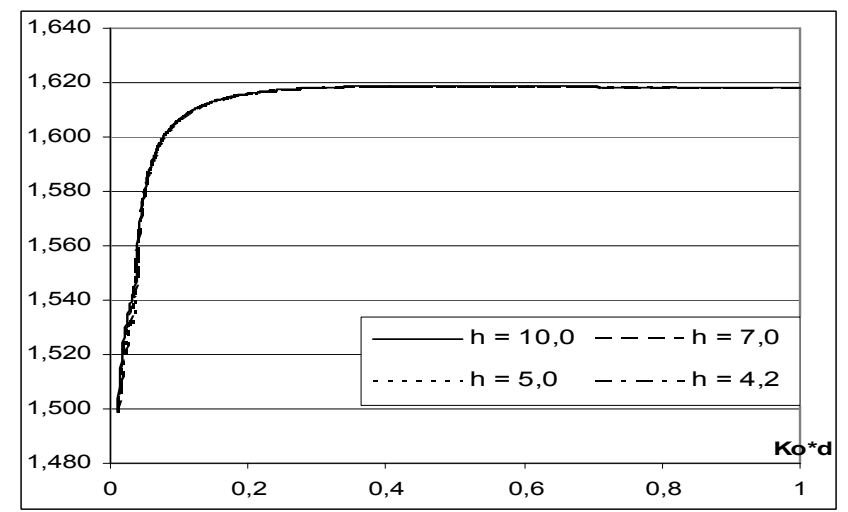

Grafico 3.c - $\left(\eta \mathrm{f}_{\mathrm{r}} \times \mathrm{K}_{\mathrm{o}} \mathrm{d}\right)$ função de $\mathrm{h}$, entre 4,1 e 10, do modo de fuga pelo núcleo $\left(1_{n}\right)$.

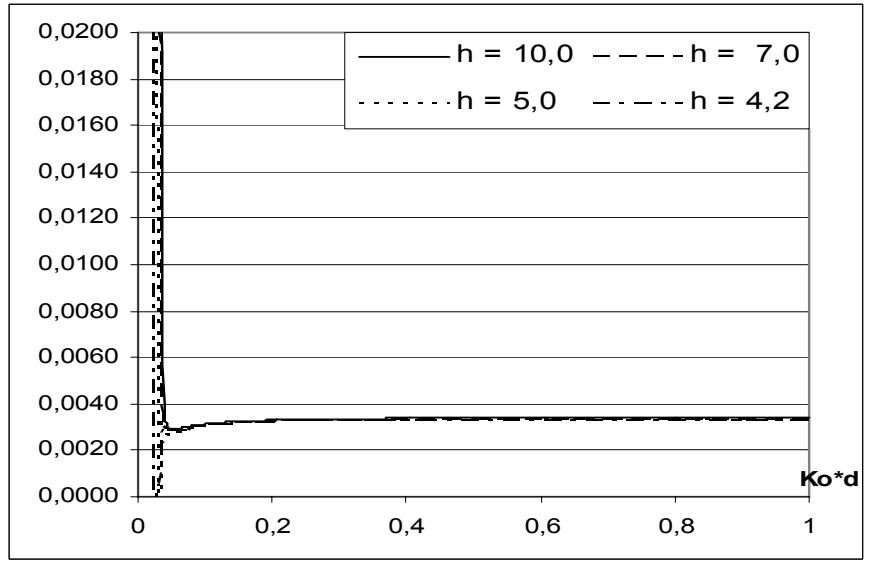

Grafico 3.d - $\left(\eta f_{\mathrm{i}} \times \mathrm{K}_{\mathrm{o}} \mathrm{d}\right)$ função de h, entre 4,1 e 10, do modo de fuga pelo núcleo $\left(l_{n}\right)$.

\section{VALIDAÇÃO DO MÉTODO}

A validação do método desenvolvido neste artigo foi feita confrontando-se os resultados obtidos, neste artigo, com os da estrutura com três regiões, publicados em [4,7]. Para isso, fezse com que a espessura do dielétrico extra sobre o filme condutor tendesse ao infinito $(\mathrm{h} \rightarrow \infty)$. Conseqüentemente, com essa consideração a estrutura se identifica com a de três regiões, uma vez que com essas dimensões, a cobertura se confunde com a região dielétrica extra $\eta 3=\eta 4=1.5$.

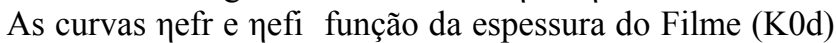
e da cobertura dielétrica extra $(\mathrm{K} 0 \mathrm{~h})$ para os modos ligados, são vistos nos gráficos 4.a e 4.b. Enquanto que, os gráficos

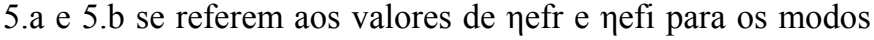
de fuga pela cobertura e pelo núcleo. Nos gráficos $5 \mathrm{a}$ e $5 \mathrm{~b}$,

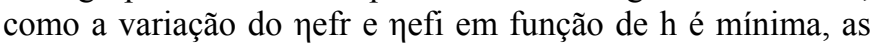
curvas de sobrepõem. Os valores assinalados nas curvas são aqueles fornecidos por $[4,7]$.

Como podem ser observados nos resultados dos gráficos, os valores confrontados entre as análises de [4,7] e os deste artigo se confundem perfeitamente.

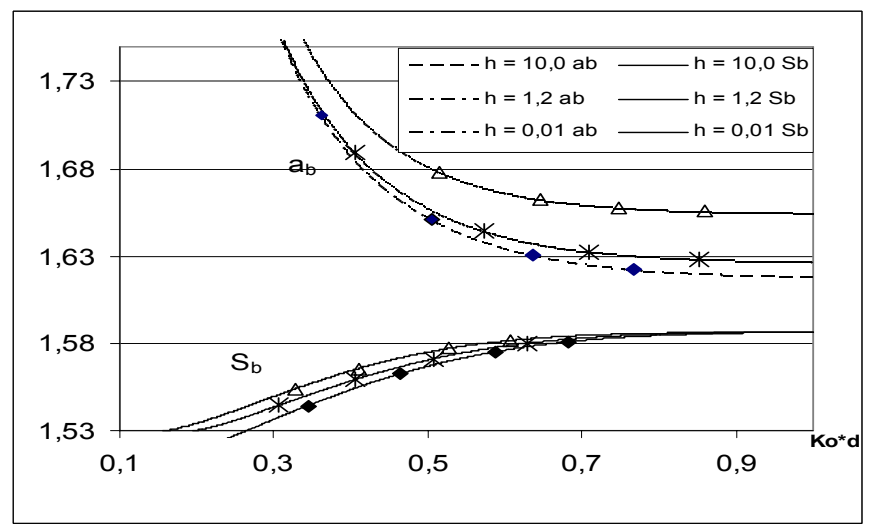

Gráfico 4.a - A parte real do índice dos modos ligados $\left(a_{b}\right.$ e $\left.S_{b}\right)$ versus a espessura normalizada do filme. 


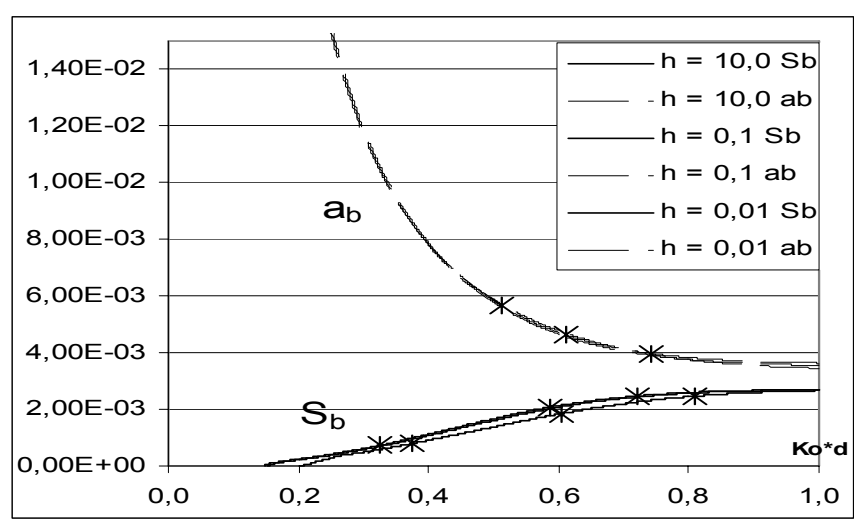

Gráfico 4.b - A parte imaginária do índice dos modos ligados $\left(\mathrm{a}_{\mathrm{b}}\right.$ e $\left.\mathrm{S}_{\mathrm{b}}\right)$ versus a espessura normalizada do filme.

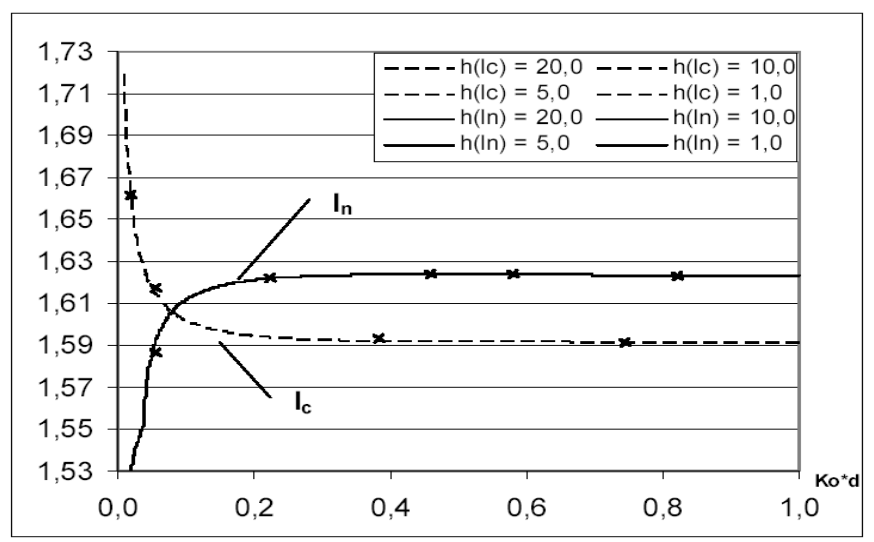

Gráfico 5.a - A parte real do índice dos modos de Fuga pelo núcleo $\left(1_{n}\right)$ e pela cobertura $\left(l_{c}\right)$ versus a espessura normalizada do filme.. As linhas sólidas representam o modo fuga pelo núcleo e as linhas pontilhadas representam o modo fuga pela cobertura.

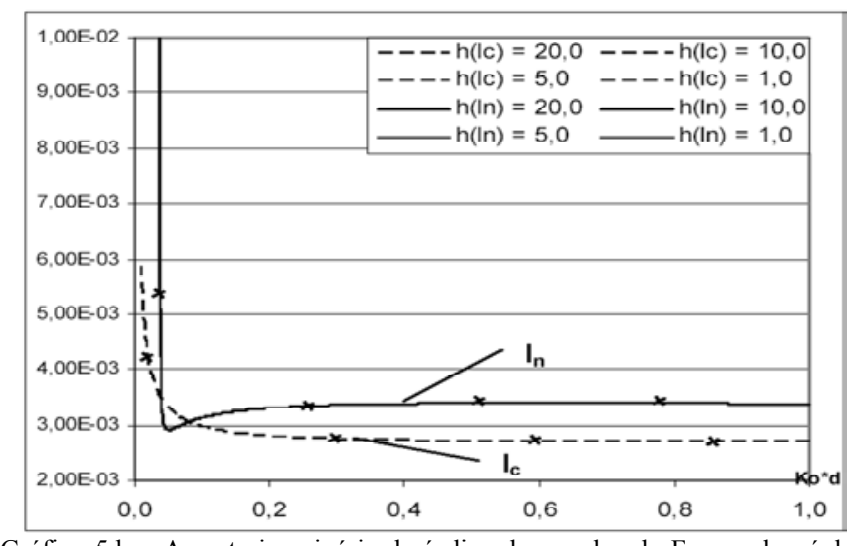

Gráfico 5.b - A parte imaginária do índice dos modos de Fuga pelo núcleo $\left(l_{n}\right)$ e pela cobertura $\left(l_{c}\right)$ versus a espessura normalizada do filme. As linhas sólidas representam o modo fuga pelo núcleo e as linhas pontilhadas representam o modo fuga pela cobertura.

\section{CONCLUSÃO}

Os resultados originais apresentados neste artigo mostraram a dependência dos modos de Plasmon com os dielétricos fronteiriços ao filme condutor, evidenciado pela presença do dielétrico extra. Destes, ficou claro que o modo de fuga pela cobertura, é o fortemente influenciado pela camada dielétrica extra que superpõe o filme, o comportamento do ๆefr deste modo, é influenciado pela espessura da referida camada. Já os modos ligados e de fuga pelo núcleo não são descaracterizados pela presença do dielétrico extra e se comportam de forma convencional ao da estrutura constituída por três regiões[4,7]. A camada dielétrica extra, retém a energia, cuja concentração se encontra na superfície do filme condutor (o que caracteriza o plasmon), reduzindo, portanto, a incidência desta energia na região, infinita, da cobertura.

O modo de fuga pela cobertura é fundamentado pela energia que flui pela camada externa da estrutura, a cobertura. $\mathrm{O}$ dielétrico extra, concentrando uma parcela dessa energia, conseqüentemente, modifica as características do referido modo, como ficou evidenciado neste artigo. Esta estrutura é muito útil na confecção de sensores ópticos.

\section{AGRADECIMENTOS}

Os autores são muito agradecidos ao Dr. José Ricardo Bergmann (CETUC/PUC/RJ) por sua inestimável contribuição e ao Prof. Luiz Antonio Palmeira Monteiro (UVA/RJ), pelo apoio, incentivo e confiança fornecidos.

\section{REFERÊNCIAS}

[1] R. E. Collin - "Field Theory of Guided Waves" - Mac GraW-Hill Book Company, 1960, Chapter -11, pp.458

[2] A. Sapienza e F. Guimarães - "Detailed Analysis of the surface Waves Guided by a thin Metal Film based en the Transverse Ressonance Method - International Microwave and Optoelectronics Conference (IMOC - 2005). Publicação em software do IMOC-2005.

[3] S.J. Al-Bader and M. Intar, "Azimuthally uniform surface plasma modes in thin metallic cylindrical shell”, IEEE, Journal of Quantum Electronics, Vol.28, no 2, pp. 525-533, February 1992.

[4] S.J. Al-Bader and M. Intar, "TM polarized modes on metal coated dielectric cylinders", Journal of Lightwave Tecnology, Vol.10, no 7, pp. 865-872, July 1992.

[5] J.J Burke, G. I. Stegeman and T. Tamir, "Surface - Polariton - like waves guided by thin, lossy metal films" - Physical Review-B, volume 33, number z, 1986, pp:5186-5201

[6] Marcelo F. Guimarães e Antonio Sapienza, "Detailled analysis of the surface plasmon waves guided by a thin metal film based in the transverse ressonance method" - Proceeding SBMO - 2005 - IEEEMTT International microwaves and optoelectronic conference, Vol.1, pp:162, 167, July 2005.

[7] Rafael A. N. Rocha, Jhonatan P. Farias, Flávia S. Ferrari e Antonio Sapienza - Análise de Plasmon em um filme metálico cobrindo uma fibra óptica fracamente guiada - Projeto de Graduação - UERJ - Junho-2007. 